\title{
Efficacy of Low Intensity Laser Therapy for Carpal Tunnel Syndrome: Experience of 54 Cases in Bangladesh
}

\author{
Farida Khatun Chhobi ${ }^{1}$, Md. Mamunul Abedin², Bijoya Dey Happy ${ }^{3}$, Md. Abdullah Yusuf ${ }^{4}$, \\ Mohammad Enayet Hussain ${ }^{5}$, Rafiqul Islam ${ }^{6}$ \\ ${ }^{1}$ Associate Professor, Department of Physical Medicine \& Neurorehabilitation, National Institute of Neurosciences \& Hospital, \\ Dhaka, Bangladesh; ${ }^{2}$ Medical Officer, Department of Physical Medicine \& Neurorehabilitation, National Institute of \\ Neurosciences \& Hospital, Dhaka, Bangladesh; ${ }^{3}$ Assistant Professor, Department of Physical Medicine \& Neurorehabilitation, \\ National Institute of Neurosciences \& Hospital, Dhaka, Bangladesh; ${ }^{4}$ Associate Professor, Department of Microbiology, \\ National Institute of Neurosciences \& Hospital, Dhaka, Bangladesh; ${ }^{5}$ Associate Professor, Department of Neurology, \\ National Institute of Neurosciences \& Hospital, Dhaka, Bangladesh; ${ }^{6}$ Associate Professor, Department of \\ Pharmacology, National Institute of Neurosciences \& Hospital, Dhaka, Bangladesh
}

[Received on: 22 April 2021; Accepted on: 12 May 2021; Published: 1 July 2021]

\begin{abstract}
Background: Management of the carpal tunnel syndrome (CTS) is very crucial for the clinical outcomes of the patients. Objective: The purpose of the present study was to assess the effectiveness of low intensity laser therapy (LILT) for the management of carpal tunnel syndrome patients. Methodology: This single centered, parallel, single blinded randomized control trial was conducted in the Department of Physical Medicine and Rehabilitation at National Institute of Neurosciences \& Hospital, Dhaka, Bangladesh from January 2019 to June 2019 for a period of six months. All the Nerve Conduction Study (NCS) test confirmed cases of CTS patients with the age group of more than 18 years in both male and female were selected as study population. Patients who received regular nonsteroidal anti-inflammatory drugs or corticosteroids, patients treated with therapeutic modalities before, acute wrist trauma and surgery, cervical radiculopathy, thoracic outlet syndrome, tumor or infectious diseases, pregnancy, and any serious heart, liver or kidney diseases were excluded from this study. Patients were randomly assigned into two groups designed as group A and group B. The assignment was done with single blind method. Group A underwent laser therapy (3 Joules $/ \mathrm{cm} 2,50 \mathrm{~Hz}, 2 \mathrm{~min}$ ) over the carpal tunnel area. Group B were treated with conventional method. All patients received therapy for a total of 14 sessions, first 7 sessions on consecutive 7 days, and last 7 sessions on alternate days. Patients also used a wrist splint each night, practiced therapeutic exercises for CTS, and followed ADL advices. Patients were assessed according to BCTQ, Boston Carpal Tunnel Questionnaire. Result: Male-Female distribution was $9 \%$ \& 91\% respectively. Total score of SSS was $26.35 \pm 5.94$ and $13.70 \pm 1.78$ in baseline and after 6 weeks follow up respectively $(\mathrm{p}<0.001)$ in group A. In group B, total score of SSS was $28.19 \pm 7.35$ and $18.22 \pm 5.63$ in baseline and after 6 weeks follow up respectively ( $<<$ 0.001). In group A, FSS was initially 18.1, which was improved to 9.15 in 6 weeks; whereas in group B, FSS was improved from 18.86 to 11.25 . The pain, numbness and paresthesia also significantly changed after 6 weeks follow up in laser therapy group. Conclusion: Low intensity laser therapy has superior clinical effectiveness at 6 weeks compared with night-resting splints in mild or moderate CTS. [Journal of National Institute of Neurosciences Bangladesh, July 2021;7(2):103-107]
\end{abstract}

\section{Keywords: Efficacy; Low Intensity; Laser Therapy; Carpal Tunnel Syndrome; LILT; LLLT; CTS}

Correspondence: Dr. Farida Khatun Chhobi, Associate Professor, Department of Physical Medicine \& Neurorehabilitation, National Institute of Neurosciences \& Hospital, Dhaka, Bangladesh; Email: fkchhobi@yahoo.com; Cell no.: +8801745407945

Conflict of interest: There is no conflict of interest relevant to this paper to disclose.

Funding agency: This research project was not funded by any group or any institution.

Contribution to authors: Chhobi FK, Abedin MM, Happy BD were involved in protocol preparation, data collection and literature search and manuscript writing. Yusuf MA, Hussain ME, Islam R were involved in preparation and revision of this manuscript.

How to cite this article: Chhobi FK, Abedin MM, Happy BD, Yusuf MA, Hussain ME, Islam R. Efficacy of Low Intensity Laser Therapy for Carpal Tunnel Syndrome: Experience of 54 Cases in Bangladesh. J Natl Inst Neurosci Bangladesh, 2021;7(2): 103-107 Copyright: (C2021. Chhobi et al. Published by Journal of National Institute of Neurosciences Bangladesh. This article is published under the Creative Commons CC BY-NC License (https://creativecommons.org/licenses/by-nc/4.0/). This license permits use, distribution and reproduction in any medium, provided the original work is properly cited, and is not used for commercial purposes.

\section{Introduction}

Carpal tunnel syndrome is the most common compression neuropathy affecting the upper limb, which results from entrapment of the median nerve in the carpal 
tunnel ${ }^{1}$. In a primary care population, prevalence has been reported to be 36.08 per 10000 people, with an annual incidence of 19.12 per 10000 for men and 35.95 per 10000 for women ${ }^{2}$. Classically, carpal tunnel syndrome causes discomfort, paresthesia, and numbness in the sensory distribution of the median nerve first 3 fingers and the radial side of the ring finger and thenar muscle weakness or atrophy. Nocturnal symptoms are often clinically significant causing sleep disturbance. The general trend of this condition is progressive worsening, although spontaneous regression of symptoms is possible, and this condition is very often misinterpreted as other conditions, like, cervical radiculopathy, general diabetic peripheral neuropathy.

Carpal tunnel syndrome is routinely classified as mild, moderate, or severe, particularly in relation to the findings of electrophysiological tests. Most cases of CTS are idiopathic. A portion of incidences of CTS are caused by pathological changes in structures passing through the carpal tunnel or the tissues forming its walls. Carpal Tunnel Pressure is raised by either decrease in Tunnel size like wrist fracture/ bony growth or increase in Content size like swelling or inflammation of tendons. This pressure rise inside the tunnel causes compression of the median nerve, altering its irrigation. The compression of the components within the carpal tunnel induces venous congestion and epineural edema. The epineural edema and the endoneural compression interrupt the axoplasmic flow of nutrients and ions leading to axonal degeneration of Median nerve \& neuritis. On the other hand, venous congestion and epineural edema consequently induces fibroblast invasion in the affected tissue causing constriction and fibrosis of the endoneural compartment of the median nerve, in conjugation with the persistency or addition of the aetiological factors which lead to chronic Carpal Tunnel Syndrome. Sub-synovial connective tissue may be predisposed to shear injury from activity done in $60^{\circ}$ of wrist flexion ${ }^{3}$. This leads to synovial tissue chronic inflammation and thickening that eventually results in idiopathic CTS which actually is due to overuse.

There are various treatment options and conservative treatment, including wrist splinting, nonsteroidal anti-inflammatory drugs (NSAIDs), and steroid injections(3). In severe CTS, surgery is often recommended. LASER (Light Amplification by Stimulated Emission of Radiation) is a device that emits light through a process of optical amplification based on the stimulated emission of electromagnetic radiation. Lasers with an output power of less than $500 \mathrm{~mW}$ or 0.5 Watts is classed as Low Intensity Laser Therapy, LILT (class III in the USA) whereas output power more than that are termed High Power Laser Therapy, HPLT (Class IV lasers in the USA). HPLT creates heat on the surface of the skin due to their higher power density (irradiance). LILT is often referred to as "Cold Lasers" since they do not create a heating sensation during treatment. Low intensity laser therapy is used to treat various musculoskeletal condition. LASER reduces pain, oedema and soothens the nerve by anti-inflammatory effects ${ }^{4}$, by selective inhibition of nociceptive activa $n$ at peripheral nerves ${ }^{5}$ by increasing ATP production and cellular respiration $^{6}$, and improvement of blood circulation to remove algesic substances ${ }^{4}$. In physiotherapy LILT is use for pain relief, accelerated tissue regeneration and reduction of inflammation. LASERs with different wavelengths, varying from 632 to $904 \mathrm{~nm}$, are used in the treatment of musculoskeletal disorders?

The mechanism of action of LASER therapy makes it a beautiful match as a therapeutic modality for pathology of CTS. But the dosimetry and the site of the points of application - We couldn't find these two things well destined in single study. Thus it was aimed for this study with a dose of 3 Joule $/ \mathrm{cm}^{2}$ at $50 \mathrm{~Hz}$ for $2 \mathrm{~min}$ for 14 sessions. Therefore, this present study was undertaken to assess the effectiveness of low intensity laser therapy for the management of carpal tunnel syndrome patients.

\section{Methodology}

Study Design \& Population: This single centered, parallel, single blinded randomized control trial was conducted in the Department of Physical Medicine and Neurorehabilitation at the National Institute of Neurosciences \& Hospital, Dhaka, Bangladesh from January 2019 to June 2019 for a period of six months. All the Nerve Conduction Study (NCS) test confirmed 54 cases of CTS patients with the age group of more than 18 years in both male and female were selected as study population. Patients who received regular non-steroidal anti-inflammatory drugs (NSAIDs) or corticosteroids, patients treated with therapeutic modalities before, acute wrist trauma and surgery, cervical radiculopathy, thoracic outlet syndrome, tumor or infectious diseases, pregnancy, and any serious heart, liver or kidney diseases were excluded from this study. Randomization \& Blinding: Patients were randomly assigned into two groups designed as group A and group $\mathrm{B}$. The assignment was done with single blind method.

Intervention: Group A underwent laser therapy (3 Joules $/ \mathrm{cm}^{2}, 2 \mathrm{~min}, 50 \mathrm{~Hz}$ ) over the carpal tunnel area. 
Group B were treated with conventional method. Among all patients, 32 received conventional therapy and 22 received laser therapy.

Follow up \& Outcome measures: All patients received therapy for a total of 14 sessions, first 7 sessions on consecutive 7 days and, last 7 sessions on alternate days. Every patient was assessed according to Boston Carpal Tunnel Questionnaire (BCTQ) before starting therapy and followed up every 2 weekly up to 6 weeks and at 12 weeks. Symptom severity scale (SSS), Pain score, Numbness, Paresthesia and Functional status scale (FSS) was measured at every follow up. Patients also used a wrist splint each night, practiced therapeutic exercises for CTS, and, followed ADL advices. Patients were assessed according to BCTQ, Boston Carpal Tunnel Questionnaire.

Statistical Analysis: Analyses were performed with SPSS software, versions 22.0 (IBM SPSS Statistics for Windows, Version 22.0. Armonk, NY: IBM Corp.). Continuous data that were normally distributed were summarized in terms of the mean, standard deviation, median, minimum, maximum and number of observations. Skewed data were presented in terms of the maximum, upper quartile, median, lower quartile, minimum and number of observations. Categorical or discrete data were summarized in terms of frequency counts and percentages. For end points analysis, Chi-square test corrected by Fisher's exact test was used for categorical variables and an analysis of variance (Student $\mathrm{t}$ Test) for continuous outcomes. A two-sided P value of less than 0.05 was considered to indicate statistical significance.

\section{Results}

A total number of 54 patients were recruited for this study. Out of 54 patients, 49 cases were female and 5 cases were male (Figure I).

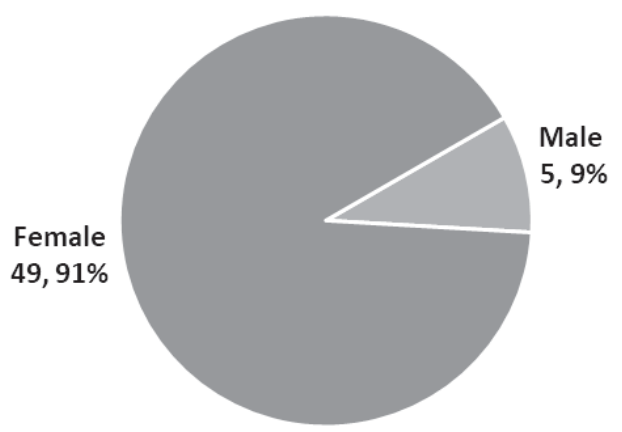

Figure 1: Sex distribution of study population
Total score of symptom severity scale (SSS) was $26.35 \pm 5.94$ and $13.70 \pm 1.78$ in baseline and after 6 weeks follow up respectively $(\mathrm{p}<0.001)$ in group A. Whereas, in group B, total score of SSS was $28.19 \pm 7.35$ and $18.22 \pm 5.63$ in baseline and after 6 weeks follow up respectively ( $<<0.001$ ) (Figure II).

Changes in Symptom Severity Scale within study population

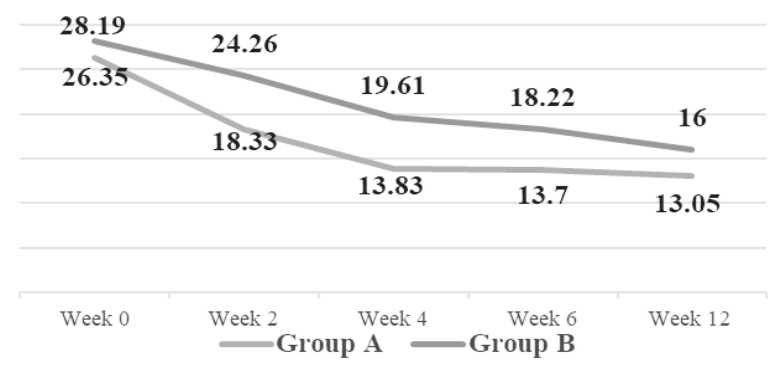

Figure II: Changes in Symptom Severity Scale within study population

Pain score $(\mathrm{p}<0.001)$, Numbness $(\mathrm{p}<0.001)$ and Paresthesia $(p<0.001)$ were also significantly reduced after 6 week follow up in both group (Figure III, IV, V).

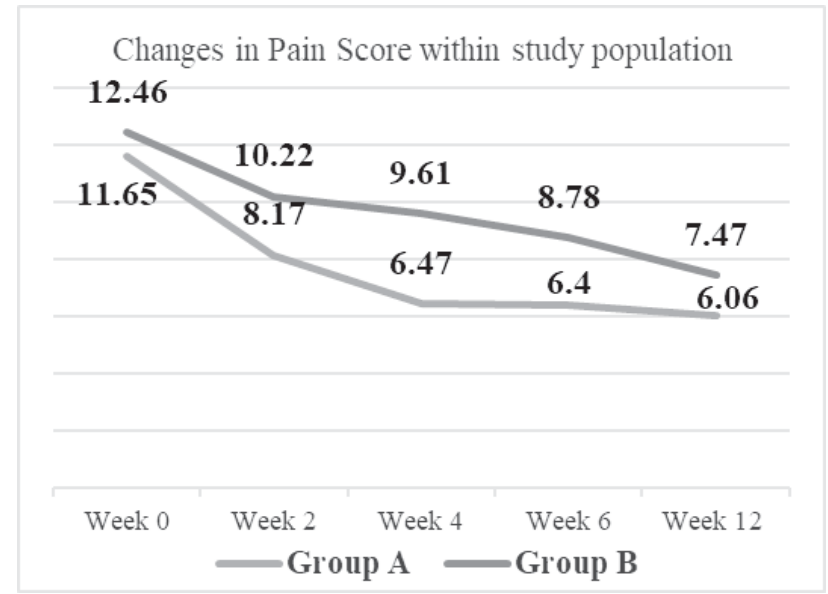

Figure III: Changes in Pain score within study population

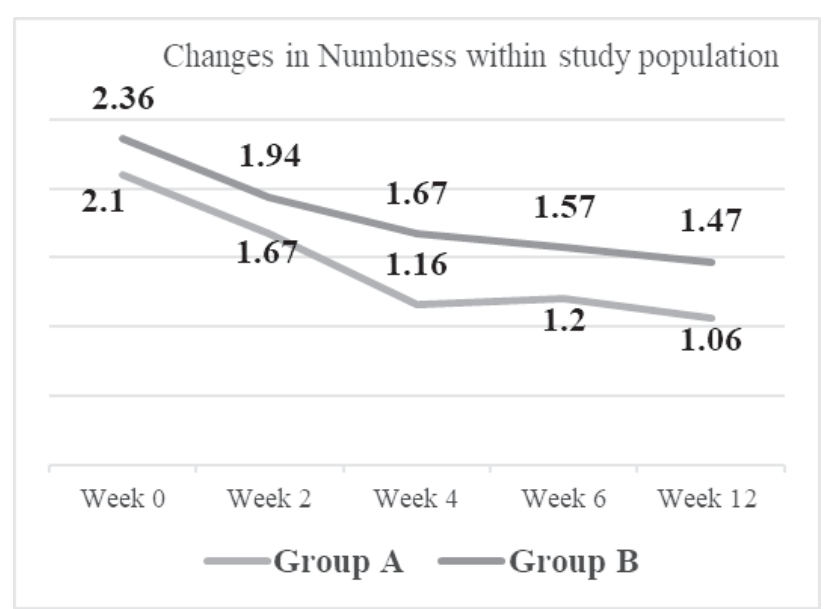

Figure IV: Changes in Numbness within study population 
Changes in Paresthesia within study population

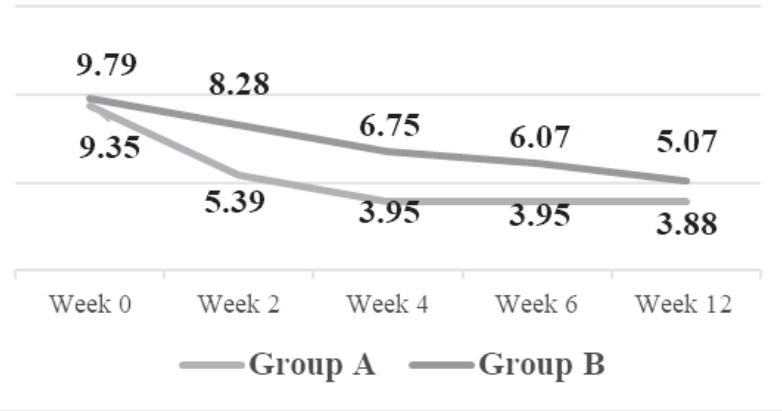

Figure V: Changes in Paresthesia within study population

In group A, FSS was initially 18.1, which was improved at 9.15 in 6 weeks; whereas in group B, FSS was improved from 18.86 to 11.25 respectively. FSS score was also changed significantly between in group $\mathrm{A}$ and $\mathrm{B}(\mathrm{p}=0.005)$ (Figure VI).

Changes in FSS within study population

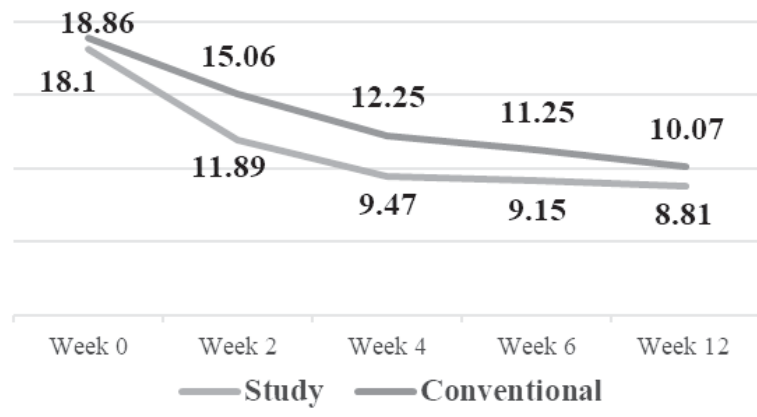

Figure VI: Changes in FSS within study population

\section{Discussion}

Previous study from other countries have demonstrated that the effectiveness of low intensity laser treatment (LILT) for CTS in comparison to the standard open carpal tunnel release surgery have overall significant results but at a lower level in relation to surgery. LILT showed significant outcomes in all parameters of subjective complaints $(\mathrm{p} \leq 0.01)$ except for muscle weakness. LILT has proven to be an effective and noninvasive treatment modality for CTS especially for early and mild-to-moderate cases when pain is the main presenting symptom ${ }^{8}$.

Kinesiotaping and low power laser in the treatment of CTS does not provide any additional benefit to the low power laser treatment in the short term but in the long term it increase the hand grip strength and finger pinch strength ${ }^{9}$. Another study of CTS with a diode laser suggest alleviating pain and symptoms and improving functional ability, and finger \& hand strength for mild and moderate CTS patient with no side effect ${ }^{10}$. The tendon gliding exercises combined with LILT or ultrasound in CTS reveals, it has beneficial effect in LILT or ultrasound combined with gliding exercise in patient with mild to moderate CTS ${ }^{11}$. However only laser acupuncture - a new non-invasive therapy which uses LILT in acupuncture could help to manage CTS ${ }^{12}$. Some article showed surgical approach has proved to be more efficient in relation to the conservative methods of steroid injection and splinting ${ }^{13}$. More high quality research is still necessary to determine the effectiveness of LILT with CTS. Only small randomized, single blind trial conducted, which is not enough to provide an evidence based assessment of the effect of LILT on CTS. Further high quality research is necessary with larger sample size and double blind study. It is possible to conclude that LILT reduces the signs and symptoms of the patients who has moderate CTS as well as the steroid treatment ${ }^{14}$. Another study revealed that LILT improved hand grip, VAS, SNAP after 3 months of follow up for mild to moderate $\mathrm{CTS}^{15}$.

Treatment Options for CTS, and LASER: There are various treatment options and conservative treatment, including wrist splinting, nonsteroidal anti-inflammatory drugs (NSAIDs), and steroid injections ${ }^{3}$. In severe CTS, surgery is often recommended. LASER (Light Amplification by Stimulated Emission of Radiation) is a device that emits light through a process of optical amplification based on the stimulated emission of electromagnetic radiation. Lasers with an output power of less than $500 \mathrm{~mW}$ or 0.5 Watts is classed as Low Intensity Laser Therapy, LILT (class III in the USA) whereas output power more than that are termed High Power Laser Therapy, HPLT (Class IV lasers in the USA). HPLT creates heat on the surface of the skin due to their higher power density (irradiance). LILT is often referred to as "Cold Lasers" since they do not create a heating sensation during treatment. Low intensity laser therapy (LILT) is used to treat various musculoskeletal condition. LASER reduces pain, oedema and soothens the nerve by anti-inflammatory effects ${ }^{4}$, by selective inhibition of nociceptive activation at peripheral nerves ${ }^{5}$, by increasing ATP production and cellular respiration ${ }^{6}$, and improvement of blood circulation to remove algesic substances ${ }^{4}$. In physiotherapy, LILT is used for pain relief, accelerated tissue regeneration, and reduction of inflammation. LASERs with different wavelengths, varying from 632 to $904 \mathrm{~nm}$, are used in the treatment of musculoskeletal disorders?

In Bangladesh, in case of carpal tunnel syndrome, the popular therapeutic modality is ultrasound therapy or 
steroid injection. Moreover, in case of application of ultrasound, physiotherapist has to hold the probe and to keep moving the probe continuously, so this is not comfortable for the applicator, this leads to their exhaustion and pain in his or her wrist after several applications. Another important thing is, in these cases, application of laser therapy takes much less time like 2 to 3 minutes only per patient where ultrasound therapy requires around 7 minutes. Low intensity laser therapy is a non-expensive and easy to apply treatment with superior clinical effectiveness at 6 weeks compared with night-resting splints in mild or moderate CTS.

\section{Conclusion}

In conclusion, the total score of symptom severity scale (SSS) has significantly decreased after 6 weeks follow up in both group A and group B. Again the clinical symptoms like pain score, numbness and paresthesia are also significantly reduced after 6 week follow up in both group A and B. FSS score was also changed significantly between in group A and B. A large scale multi-center study should be conducted to see the real scenario.

\section{References}

1. Aroori S, Spence RAJ. Carpal tunnel syndrome. Ulster Med J 2008;77(1):6-17.

2. Burton CL, Chen Y, Chesterton LS, van der Windt DA. Trends in the prevalence, incidence and surgical management of carpal tunnel syndrome between 1993 and 2013: an observational analysis of UK primary care records. BMJ Open 2018;8(6):e020166-e020166

3. Gerritsen AAM, de Krom MCTFM, Struijs MA, Scholten RJPM, de Vet HCW, Bouter LM. Conservative treatment options for carpal tunnel syndrome: a systematic review of randomised controlled trials. J Neurol. 2002;249(3):272-80.

4. Honmura A, Ishii A, Yanase M, Obata J, Haruki E. Analgesic effect of Ga-Al-As diode laser irradiation on hyperalgesia in carrageenin-induced inflammation. Lasers Surg Med. 1993;13(4):463-9.

5. Jarvis D, MacIver MB, Tanelian DL. Electrophysiologic recording and thermodynamic modeling demonstrate that helium-neon laser irradiation does not affect peripheral $\mathrm{A} \delta$ - or C-fiber nociceptors. Pain. 1990;43(2):235-42.

6. Passarella S. He-Ne laser irradiation of isolated mitochondria. Vol. 3, Journal of photochemistry and photobiology. B, Biology. Switzerland; 1989. p. 642-3.

7. Yousefi-Nooraie R SEHKRAPVAMISSBMHSJAR, MortazHedjri S. Low level laser therapy for nonspecific low-back pain. Cochrane Database Syst Rev [Internet]. 2008;(2).

8. Elwakil T, Elazzazi A, Shokeir H. Treatment of carpal tunnel syndrome by low-level laser versus open carpal tunnel release. Lasers Med Sci. 2007;22:265-70.

9. Güner A, Altan L, Kasapoğlu Aksoy M. The effectiveness of the low-power laser and kinesiotaping in the treatment of carpal tunnel syndrome, a pilot study. Rheumatol Int. 2018 May 1;38.

10. Chang W-D, Wu J-H, Jiang J-A, Yeh C-Y, Tsai C-T. Carpal Tunnel Syndrome Treated with a Diode Laser: A Controlled Treatment of the Transverse Carpal Ligament. Photomed Laser Surg. 2008;26:551-7.

11. Bartkowiak Z, Eliks M, Zgorzalewicz-Stachowiak M, Romanowski L. The Effects of Nerve and Tendon Gliding Exercises Combined with Low-level Laser or Ultrasound Therapy in Carpal Tunnel Syndrome. Indian J Orthop. 2019;53:347.

12. Chen C-C, Wu Y-T, Su Y-C, Shen Y-P, Chen F-P. Efficacy of laser acupuncture for carpal tunnel syndrome: A study protocol for a prospective double-blind randomized controlled trial. Medicine (Baltimore). 2019;98(30):e16516.

13. Kuddus MR, Faruk MO, Alam S, Islam KA, Alam S, Debnath H. Management of Carpal Tunnel Syndrome - Surgical Vs Medical? Bangladesh J Neurosurg. 2020;10(1):75-81.

14. Badıl Gülo\&amp;amp;gbreve;lu S, Bilgilisoy Filiz M, Kılıç KK, Koldaş Do\&amp;amp;gbreve;an S, Erdem Toslak I, Toraman NF. THU0543 Comparison of the effectiveness of low level laser therapy and steroid injection in moderate carpal tunnel syndrome: a randomised controlled trial. Ann Rheum Dis [Internet]. 2018;77(Suppl 2):474 LP - 474

15. Li Z-J, Wang Y, Zhang H-F, Ma X-L, Tian P, Huang Y. Effectiveness of low-level laser on carpal tunnel syndrome: A meta-analysis of previously reported randomized trials. Medicine (Baltimore) [Internet]. 2016;95(31):e4424-e4424 